\title{
ABELIAN GROUP ALGEBRAS OF FINITE ORDER
}

\author{
BY \\ SAM PERLIS AND GORDON L. WALKER
}

Introduction. A group $G$ of finite order $n$ and a field $F$ determine in well known fashion an algebra $G_{F}$ of order $n$ over $F$ called the group algebra of $G$ over $F$. One fundamental problem( ${ }^{(1)}$ is that of determining all groups $H$ such that $H_{F}$ is isomorphic to $G_{F}$.

It is convenient to recast this problem somewhat: If groups $G$ and $H$ of order $n$ are given, find all fields $F$ such that $G_{F}$ is isomorphic to $H_{F}$ (notationally: $G_{F} \cong H_{F}$ ). We present a complete solution of this problem for the case in which $G$ (and thus necessarily $H$ ) is abelian and $F$ has characteristic infinity or a prime not dividing $n$. The result, briefly, is that $F$ shall contain a certain subfield which is determined by the invariants of $G$ and $H$ and the characteristic of $F$.

1. Multiplicities. If $G$ is abelian of order $n$ and $F$ is a field whose characteristic does not divide $n$, the group algebra $G_{F}$ has the structure

$$
G_{F}=\sum_{d \mid n} a_{d} F\left(\zeta_{d}\right)
$$

where $\zeta_{d}$ is a primitive $d$ th root of unity, $a_{d}$ is a non-negative integer, and $a_{d} F\left(\zeta_{d}\right)$ denotes the direct sum of $a_{d}$ isomorphic copies of $F\left(\zeta_{d}\right)$. In fact, each irreducible representation $S$ of $G_{F}$ maps $G_{F}$ onto a field $F_{S} \geqq F$ and maps the elements of $G$ on $n$th roots of unity. The image of $G$ is a subgroup of the group of all $n$th roots of unity, thus is a cyclic group of some order dividing $n$. It follows that $F_{S}=F\left(\zeta_{d}\right)$ where $\zeta_{d}$ is a primitive $d$ th root of unity. Formula (1) expresses the fact that a complete set of irreducible representations of $G_{F}$ over $F$ include precisely $a_{d}$ which map $G$ onto a cyclic group of order $d$. Now if $K$ is the root field over $F$ of $x^{n}-1=0$ we have

$$
G_{K}=\sum_{d \mid n} n_{d} K_{d}
$$

where every $K_{d}=K\left(\zeta_{d}\right)$ is isomorphic to $K, \sum n_{d}=n$, and each $n_{d}$ is the number of irreducible representations $T$ of $G_{K}$ mapping $G$ on a cyclic group of order $d$.

LEMMA 1. The integer $n_{d}$ in (2) is the number of elements of order $d$ in $G$.

There is a one-to-one correspondence between the elements $g$ of $G$ and the

Presented to the Society, April 16, 1948, and October 30, 1948, under the titles Finite abelian group algebras, I and II ; received by the editors September 22, 1948 and, in revised form, April 29, 1949.

(1) Proposed by R. M. Thrall at the Michigan Algebra Conference in the summer of 1947. 
representations $T=T_{g}$. The formulae $\left({ }^{2}\right)$ for this correspondence make it evident that $g$ has order $d$ if and only if $T_{g}$ maps a basis of $G$ onto a set of elements, the l.c.m. of whose orders is $d$. Then some element of $G$ is mapped on an element of order $d$, all others on elements of order not greater than $d$. The map of $G$ is thus a cyclic group of order $d$, and this proves the lemma.

Each irreducible representation $S$ of $G_{F}$ over $F$ may be extended to a representation of $G_{K}$ over $K$, the extension not altering the map of $G$. If $S$ maps $G_{F}$ onto $F\left(\zeta_{d}\right)$ where the degree of $F\left(\zeta_{d}\right) / F$ is

$$
\operatorname{deg} F\left(\zeta_{d}\right) / F=v_{d},
$$

then $S$ maps $G_{K}$ on the direct $\operatorname{sum}\left({ }^{3}\right)$

$$
F\left(\zeta_{d}\right)_{K}=K^{(1)} \oplus \cdots \oplus K^{\left(v_{d}\right)}=v_{d} K,
$$

thus giving rise to $v_{d}$ irreducible representations $T$ of $G_{K}$ over $K$.

LEMMA 2. If $S$ maps $G$ onto a cyclic group of order $d$, so does each representation $T$ defined above.

Each element $g$ in $G$ is mapped by $S$ on $g^{S}=\sum g_{i}, g_{i}$ in $K^{(i)}$, and the corresponding irreducible representations over $K$ are $T_{i}: g^{T_{i}}=g_{i}$. It may be seen $\left({ }^{4}\right)$ that the $g_{i}$ are obtainable from one another by automorphisms of $F\left(\zeta_{d}\right)_{K}$ leaving the elements of $K$ invariant. Hence all the $g_{i}$ have the same minimum function over $K$, and all of them are primitive $d$ th roots of unity if $g S$ is one. Lemma 2 follows immediately, and it follows that the $T_{i}$ into which the representations $S$ split are the only irreducible representations of $G_{K}$ mapping $G$ on a cyclic group of order $d$. The $a_{d}$ choices of $S$ give rise to $a_{d} v_{d}$ representations $T$, whence $n_{d}=a_{d} v_{d}$.

TheOREM 1. The multiplicity $a_{d}$ in (1) is given $\left(^{5}\right)$ by $a_{d}=n_{d} / v_{d}$ where $n_{d}$ is the number of elements of order $d$ in $G$ and $v_{d}$ is $\operatorname{deg} F\left(\zeta_{d}\right) / F$.

Now let $G$ and $H$ be abelian of common order $n=p_{1}^{\theta_{1}} \cdots p_{k}^{k_{k}}$ for distinct primes $p_{i}$, so there are unique expressions $G=G_{1} \times \cdots \times G_{k}$ and $H=H_{1}$ $\times \cdots \times H_{k}$ for $G$ and $H$ as direct products of groups $G_{i}$ and $H_{i}$ of order $n_{i}=p_{i}^{i_{i}}$. Then:

COROLLARY 1. $G_{F} \cong H_{F}$ if and only if $G_{i F} \cong H_{i F}$ for $i=1, \cdots, k$.

By hypothesis and Theorem 1

(2) A. Speiser, Die Theorie der Gruppen von endlicher Ordnung, New York, 1945, p. 179.

(3) A. A. Albert, Structure of algebras, Amer. Math. Soc. Colloquium Publications, vol. 24, New York, 1939, p. 31.

(4) Ibid.

(5) The authors are indebted to the referees for the simple approach to Theorem 1 which has been presented here. 


$$
\begin{gathered}
G_{F}=\sum_{d \mid n} m_{d} / v_{d} F\left(\zeta_{d}\right) \cong H_{F}, \\
G_{i F}=\sum_{d \mid n_{i}} g_{i d} / v_{d} F\left(\zeta_{d}\right), \quad H_{i F}=\sum_{d \mid n_{i}} h_{i d} / v_{d} F\left(\zeta_{d}\right)
\end{gathered}
$$

where the number of elements of order $d$ in $G_{i}$ is $g_{i d}$, in $H_{i}$ is $h_{i d}$, and in $G$ or $H$ is $m_{d}$. But if $d \mid n_{i}$, the elements of $G$ having order $d$ lie in $G_{i}$, so $m_{d}=g_{i d}$ and likewise $m_{d}=h_{i d}$ so $g_{i d}=h_{i d}$, whence $G_{i F} \cong H_{i F}$. The converse is trivial.

In the remaining sections only the prime-power case is considered.

2. Cyclotomic fields. When $n=p^{\alpha}$ for a prime $p$ the notation in (1) will be changed to

$$
G_{F}=\sum_{i=0}^{\alpha} a_{i} F\left(\zeta_{i}\right)
$$

where $\zeta_{i}$ and $a_{i}$ are new symbols for $\zeta_{d}$ and $a_{d}, d=p^{i}$. This section explores conditions under which $F\left(\zeta_{i}\right) \cong F\left(\zeta_{j}\right)$. Taking $i \leqq j$ we may and shall assume that $F\left(\zeta_{i}\right) \leqq F\left(\zeta_{j}\right)$, so the question now is concerned with the equality of these fields. Let $P$ always denote the prime subfield of $F$.

Leмma 3. Let $i$ and $j$ be positive integers such that $i<j$. Then $F\left(\zeta_{i}\right)=F\left(\zeta_{j}\right)$ if and only if $F$ has a subfield $F_{0} \leqq P\left(\zeta_{j}\right)$ such that $F_{0}\left(\zeta_{i}\right)=F_{0}\left(\zeta_{j}\right)$.

Proof. If $F_{0}\left(\zeta_{i}\right)=F_{0}\left(\zeta_{j}\right)$, the field $F\left(\zeta_{i}\right)$ must contain $\zeta_{j}$. Conversely, suppose $F\left(\zeta_{i}\right)=F\left(\zeta_{j}\right)$. The minimum function $f(x)$ of $\zeta_{j}$ over $F$ has degree $s$ equal to that of $\zeta_{i}$, and is a factor of the minimum function $m(x)$ of $\zeta_{j}$ over $P$. The coefficients of $f(x)$ then must lie in the root field $P\left(\zeta_{j}\right)$ of $m(x)$ over $P$, and hence generate a subfield $F_{0}$ of $P\left(\zeta_{j}\right)$ such that $F_{0} \leqq F$. Then $F_{0}\left(\zeta_{j}\right) \geqq F_{0}\left(\zeta_{i}\right)$, and

$$
\operatorname{deg} F_{0}\left(\zeta_{j}\right) / F_{0}=s \geqq \operatorname{deg} F_{0}\left(\zeta_{i}\right) / F_{0}=r \geqq \operatorname{deg} F\left(\zeta_{i}\right) / F=s,
$$

whence $r=s, F_{0}\left(\zeta_{i}\right)=F_{0}\left(\zeta_{j}\right)$.

It is necessary now to make a brief detour because of some peculiarities arising if $P$ is finite. Suppose that

$$
P \leqq P\left(\zeta_{1}\right)=\cdots=P\left(\zeta_{e}\right)<P\left(\zeta_{e+1}\right)
$$

if $p$ is odd, and

$$
P \leqq P\left(\zeta_{2}\right)=\cdots=P\left(\zeta_{e}\right)<P\left(\zeta_{e+1}\right)
$$

if $p=2$. These equalities never occur if $P=R$ but do occur if $P$ is a finite prime field whose characteristic is appropriately related to $p$ (see Lemma 5).

Definition. Let $p$ be a prime and let $P$ be a prime field of characteristic not equal to $p$. Then the integer $e$ defined by (6) and (7) is called the cyclotomic number of $P$ relative to $p$ (or cyclotomic $p$-number of $P$ ).

Lemma 4. Let $P$ be a finite prime field of characteristic $\pi, n$ be an integer not 
divisible by $\pi$, and $P(\zeta)$ be the root field over $P$ of $x^{n}-1$. Then $\operatorname{deg} P(\zeta) / P=\epsilon$ where $\epsilon$ is defined as the exponent to which $\pi$ belongs modulo $n$.

Let $P_{f}$ be a field of degree $f$ over $P$ so its nonzero quantities are roots of $x^{\nu}-1=0, \nu=\pi^{f}-1$. Then $P_{f}$ contains the $n$th roots of unity if $n$ divides $\nu$. Conversely, if $P_{f}$ contains a primitive $n$th root of unity, $\zeta$, the equation $\nu=q n+r(0 \leqq r<n)$ leads to $\zeta^{\nu}=1=\zeta^{r}$ so $r=0$, and $n$ divides $\nu$. The smallest value of $\nu=\pi^{f}-1$ obeying this condition is given by $f=\epsilon$. On the other hand the smallest value surely belongs to $P_{f}=P(\zeta)$.

Now let $n=p^{i}$, where $p$ is a prime not equal to $\pi$, and denote the corresponding integer $\epsilon$ of Lemma 4 by $\epsilon_{i}$. Then the cyclotomic $p$-number of $P$ is the integer $e$ determined by the conditions $\epsilon_{1}=\epsilon_{2}=\cdots=\epsilon_{e}<\epsilon_{e+1}$ ( $p$ odd), $\epsilon_{2}=\epsilon_{3}=\cdots=\epsilon_{e}<\epsilon_{e+1}(p=2)$. Hence:

LEMmA 5. The cyclotomic p-number of $P$ is the maximum integer e such that $p^{e}$ divides $\pi^{e}-1$ where $\epsilon$ is the exponent to which $\pi$ belongs modulo $p$ if $p$ is odd, or modulo 4 if $p=2$.

The fact that $P\left(\zeta_{i}\right)<P\left(\zeta_{i+1}\right)$ for every $i \geqq e$ is a consequence of the following result.

Lemma 6. The extension $P\left(\zeta_{e+i}\right) / P\left(\zeta_{e}\right)$ has degree $\delta_{i}=p^{i}(i=1,2, \cdots)$.

Writing $\epsilon_{e}=\epsilon$ we have $\delta_{i}=\epsilon_{e+i} / \epsilon$ and know $\left(^{6}\right)$ that $\delta_{i}=p^{j}, j \leqq i, \epsilon_{e+i}=p^{j} \epsilon$. By Lemma $5, \pi^{e}=1+a p^{e}$ where $a$ is not divisible by $p$. A trivial induction shows that

$$
\pi^{p^{i_{\epsilon}}}=1+a_{i} p^{e+i}, \quad\left(a_{i}, p\right)=1,
$$

for $i=0,1,2, \cdots$. This proves that $\epsilon_{e+i}=p^{i} \epsilon$.

Lemma 7. If $p$ is an odd prime and $P$ is any prime field of characteristic not $p, P\left(\zeta_{q}\right)$ has the structure

$$
P\left(\zeta_{q}\right)=P\left(\zeta_{1}\right) \times L_{q}, \quad \operatorname{deg} L_{q} / P=\text { power of } p,
$$

where $L_{q}$ is unique. Moreover, $L_{q}=P$ if $q$ does not exceed the cyclotomic p-number of $P$.

The proof of this result is similar to the known $\left({ }^{7}\right)$ proof for the case $P=R$.

Lemмa 8. Let $p$ be odd and $q>1$. Then the following conditions are equivalent:

(i) $F\left(\zeta_{q}\right)=F\left(\zeta_{i}\right), 1 \leqq i<q$.

(ii) $F\left(\zeta_{q}\right)=F\left(\zeta_{q-1}\right)=\cdots=F\left(\zeta_{1}\right)$.

(iii) $F$ contains the field $L_{q}$ defined by Lemma 7.

(6) A. A. Albert, Modern higher algebra, Chicago, 1937, p. 188, Theorem 21. The desired result is obtained by repeated application of this reference theorem.

(7) Robert Fricke, Lehrbuch der Algebra, vol. 3, Braunschweig, 1928, p. 205. 
The condition (iii) implies that $F\left(\zeta_{1}\right)$ contains $L_{q}\left(\zeta_{1}\right)=P\left(\zeta_{q}\right), \quad F\left(\zeta_{1}\right)$ $=F\left(\zeta_{q}\right)$, so (ii) follows. That (ii) implies (i) is obvious. Now we assume (i) and use Lemma 3 to reduce considerations to the case $F \leqq P\left(\zeta_{q}\right)=F\left(\zeta_{q}\right)$. If $q \leqq e$ where $e$ is the cyclotomic $p$-number of $P, L_{q}=P \leqq F$ so (iii) is valid. Now let $q$ be greater than $e$.

The field $F\left(\zeta_{i}\right)$ is the composite $F \cup P\left(\zeta_{i}\right)$. Denoting the intersection $F \cap P\left(\zeta_{i}\right)$ by $F_{i}$, we have

$$
\operatorname{deg} F / F_{i}=\operatorname{deg} F\left(\zeta_{i}\right) / P\left(\zeta_{i}\right)=\operatorname{deg} P\left(\zeta_{q}\right) / P\left(\zeta_{i}\right) .
$$

Also, $\operatorname{deg} P\left(\zeta_{k}\right) / P=p^{\epsilon_{k}} u, \operatorname{deg} F / P=p^{a_{v}}$ for suitable integers $\epsilon_{k}, a, u$ $=\operatorname{deg}\left(P\left(\zeta_{1}\right) / P\right.$, and $v$ a divisor of $u$. To complete preparations for substituting in (9) note that $P\left(\zeta_{q}\right) / P$ is cyclic, hence possesses a unique subfield of any given degree dividing $p^{\epsilon} q$. Thus: $\operatorname{deg} F_{i} / P=\operatorname{gcd}\left[p^{a} v, p^{\boldsymbol{e} i u}\right]=p^{\mu} v$ where $\mu=\min \left[a, \epsilon_{i}\right]$. From (9), $p^{a-\mu}=p^{c}$ where $c=\epsilon_{q}-\epsilon_{i}=a-\mu$. Since $q>e$, we have $\epsilon_{q}-\epsilon_{i}>0, \mu<a, \mu=\epsilon_{i}$, so $a=\epsilon_{q}$, deg $F / P=p^{\epsilon q v}$. Every such subfield $F$ of $P\left(\zeta_{q}\right)$ must contain the subfield $L_{q}$ of degree $p^{\epsilon q}$.

For the case $p=2$ similar results are obtainable. The extension $P\left(\zeta_{q}\right) / P$ is cyclic of degree a power of 2 if $P$ is finite, and for this case we define

$$
L_{q}=P \quad \text { if } \quad q \leqq e, \quad L_{q}=P\left(\zeta_{q}\right) \text { if } q>e,
$$

where $e$ is the cyclotomic number of $P$ relative to $p=2$. For $P=R$ we have $P\left(\zeta_{q}\right)=P\left(\zeta_{2}\right) \times L_{q}$ where $L_{q}$ is arbitrarily one of the fields

$$
L_{q}=P\left(\zeta_{q}+\zeta_{q}^{-1}\right), \quad L_{q}=P\left(\zeta_{q}-\zeta_{q}^{-1}\right)
$$

and $\operatorname{deg} L_{q} / P=2^{q-2}$. We then state without proof:

LEMMA 9. Let $p=2$ and $q>2$. Then the following conditions are equivalent:

(i) $F\left(\zeta_{q}\right)=F\left(\zeta_{i}\right), 2 \leqq i<q$.

(ii) $F\left(\zeta_{q}\right)=F\left(\zeta_{q-1}\right)=\cdots=F\left(\zeta_{2}\right)$.

(iii) $F$ contains one of the fields $L_{q}$ above.

3. Determination of the fields. Let $G$ and $H$ be abelian groups of common prime-power order $p^{\alpha}$ and let $F$ be any field of characteristic not $p$. In this section all fields $F$ are determined such that $G_{F} \cong H_{F}$.

As in (5) we have

$$
G_{F}=\sum_{i=0}^{\alpha} a_{i} F\left(\zeta_{i}\right), \quad H_{F}=\sum_{i=0}^{\alpha} b_{i} F\left(\zeta_{i}\right),
$$

so there is a unique integer $q=q(G, H)$ defined as the maximum integer $i$ such that $a_{i} \neq b_{i}$. From Theorem 1 this integer is the maximum $i$ such that $m_{i} \neq n_{i}$ where $m_{i}$ and $n_{i}$ are the numbers of elements of order $p^{i}$ in $G$ and $H$, respectively. Thus $q$ is independent of $F$. Since $m_{0}=n_{0}=1, q$ is never less than 2 , but it may happen that $q$ does not exist, that is, every $m_{i}=n_{i}$. In 
this case we define $q=0$.

THEOREM 2. The group algebras $G_{F}$ and $H_{F}$ are isomorphic if and only if $(\alpha)$ holds when $p$ is odd, and $(\beta)$ or $(\gamma)$ holds when $p=2$ :

( $\alpha) F \geqq L_{q}$ defined by Lemma 7 .

( $\beta$ ) $G$ and $H$ have the same number of invariants and $F$ contains one of the fields $L_{q}$ defined by Lemma 9.

$(\gamma) G$ and $H$ have unequal numbers, $\gamma$ and $\eta$, of invariants and $F$ contains $P\left(\zeta_{q}\right)$ where $P$ is the prime subfield of $F$.

If $q=0$ the theorem is trivial, so we assume $q>0$, hence $q \geqq 2$. Note that $G_{F} \cong H_{F}$ if and only if $A \cong B$ where

$$
A=\sum_{i=0}^{q} a_{i} F\left(\zeta_{i}\right), \quad B=\sum_{i=0}^{q} b_{i} F\left(\zeta_{i}\right) .
$$

Suppose $(\alpha)$ holds. Then (Lemma 8 ) both $A$ and $B$ becomes $F \oplus m F\left(\zeta_{1}\right)$ for a suitable integer $m$, so $A \cong B$. If $p=2, F\left(\zeta_{1}\right)=F, a_{1}=2^{\gamma}-1$ so

$$
A=2^{\gamma} F \oplus \sum_{i=2}^{q} a_{i} F\left(\zeta_{i}\right), \quad B=2^{\eta} F \oplus \sum_{i=2}^{q} b_{i} F\left(\zeta_{i}\right)
$$

whence $(\beta)$ implies that $A=2^{\gamma} F \oplus m F\left(\zeta_{2}\right) \cong B$. If $(\gamma)$ holds, $A$ and $B$ are diagonal over $F$ and of the same order, hence isomorphic. Conversely, suppose $A \cong B$ and first let $p$ be odd. The assumption that $F\left(\zeta_{q}\right)$ is not isomorphic to $F\left(\zeta_{i}\right)$ for $i<q$ implies that $A$ has precisely $a_{q}$ components $F\left(\zeta_{q}\right)$ and $B$ has precisely $b_{q}$ such components. But then the fact that $a_{q} \neq b_{q}$ conflicts with the isomorphism of $A$ and $B$. Hence $F\left(\zeta_{q}\right)=F\left(\zeta_{i}\right)$ for $i<q$ so $F \geqq L_{q}$. The proofs for $p=2$ are obtained in similar fashion.

The case in which $F$ is a prime field is interesting.

THEOREM 3. Let $G$ and $H$ be abelian groups of order $p^{\alpha}$. If $R$ is the rational number field, $G_{R} \cong H_{R}$ if and only if $G \cong H$. If $P$ is a finite prime field of characteristic $\pi \neq p, G_{P} \cong H_{P}$ if and only if $q \leqq e$ (where $e$ is the cyclotomic p-number of $P$ ) unless $p=2$ and $G$ and $H$ have different numbers of invariants. In the latter case $G_{P} \cong H_{P}$ if and only if $q \leqq e$ and $\pi \equiv 1(\bmod 4)$.

For $F=R$ the decompositions (12) are unique. Hence the condition $G_{R} \cong H_{R}$ implies that $q=0$, and for each integer $k=p^{h}$ dividing $p^{\alpha}, G$ and $H$ have the same number of elements of order $k$. This number is $N_{k}(G) \phi(k)$ where $\phi$ denotes the Euler $\phi$-function and $N_{k}(G)=N_{k}$ the number of cyclic subgroups of order $k$ in $G$. The numbers $N_{k}$ have been determined( $\left.{ }^{8}\right)$ by formulae which show that the group invariants are determined when the $N_{k}$

(8) G. A. Miller, Number of the sub-groups of any abelian group, Proc. Nat. Acad. Sci. U. S. A. vol. 25 (1939) pp. 256-262; see also Yenchien Yeh, On prime power abelian groups, Bull. Amer. Math. Soc. vol. 54 (1948) pp. 323-327. 
are specified. Thus $G \cong H$. The remaining parts of the theorem follow from Theorem 2 and our lemmas.

To compute the " $q$-number" directly from the invariants of $G$ and $H$, denote the latter by $p^{e_{i}}(i=1, \cdots, \gamma)$ and $p^{f_{i}}(i=1, \cdots, \eta)$, respectively, numbered in descending order of magnitude.

Theorem 4. Define $\lambda$ as the minimum integer $i$ such that $e_{i} \neq f_{i}$. Then $q=\max \left[e_{\lambda}, f_{\lambda}\right]$.

For proof, note that $G=K \times \bar{G}, H=K \times \bar{H}$ where $K$ has invariants $p^{e_{i}}$, $i=1, \cdots, \lambda-1$, and those of $\bar{G}$ and $\bar{H}$ are evident. Let the common order of $\bar{G}$ and $\bar{H}$ be $\bar{n}$ and let the numbers of elements of order $p^{i}$ in $G, H$, and $K$, respectively, be $m_{i}, n_{i}$, and $k_{i}$. Then $i>e_{\lambda}$ implies $m_{i}=\bar{n} k_{i}$ and $i>f_{\lambda}$ implies $n_{i}=\bar{n} k_{i}$. For definiteness take $e_{\lambda}>f_{\lambda}$, so $i>e_{\lambda}$ implies $m_{i}=n_{i}, q \leqq e_{\lambda}$. For $i=e_{\lambda}>f_{\lambda}$, however, $n_{i}=\bar{n} k_{i}, m_{i}>n_{i}$. This proves that $q=e_{\lambda}$.

PURDUE UNIVERSITY, LAFAYETTE, IND. 